\title{
Parity-time-antisymmetric atomic lattices without gain
}

\author{
Jin-Hui Wu, ${ }^{1}$ M. Artoni, ${ }^{2,3}$ and G. C. La Rocca ${ }^{4}$ \\ ${ }^{1}$ Center for Quantum Sciences, Northeast Normal University, Changchun 130117, China \\ ${ }^{2}$ European Laboratory for Nonlinear Spectroscopy (LENS), 50019 Firenze, Italy \\ ${ }^{3}$ Department of Engineering and Information Technology \& Istituto Nazionale Ottica (INO-CNR), Brescia University, 25133 Brescia, Italy \\ ${ }^{4}$ Scuola Normale Superiore and CNISM, 56126 Pisa, Italy \\ (Received 27 January 2015; published 11 March 2015)
}

\begin{abstract}
Lossy atomic photonic crystals can be suitably tailored so that the real and imaginary parts of the susceptibility are, respectively, an odd and an even function of position. Such a parity-time $(P T)$ space antisymmetry in the susceptibility requires neither optical gain nor negative refraction, but is rather attained by a combined control of the spatial modulation of both the atomic density and their dynamic level shift. These passive photonic crystals made of dressed atoms are characterized by a tunable unidirectional reflectionlessness accompanied by an appreciable degree of transmission. Interestingly, such peculiar properties are associated with non-Hermitian degeneracies of the crystal scattering matrix, which can then be directly observed through reflectivity measurements via a straightforward phase modulation of the atomic dynamic level shift and even off resonance.
\end{abstract}

DOI: 10.1103/PhysRevA.91.033811

PACS number(s): 42.50.Nn, 42.50.Gy, 11.30.Er, 37.10.Jk

\section{INTRODUCTION}

Artificial optical materials have attracted great attention in the past few decades for achieving various properties and functionalities not available in natural media. Photonic crystals [1,2] and left-handed materials [3] are two prominent metamaterials promising the possibility of stretching usual rules and displaying new paradigms of light propagation and interaction. In fact, they have been well exploited to design and realize all-optical, optoelectronic, and optomechanical devices like ultrafast light switching, diffraction free superlens, and quantum motion detectors [4-7], just to name a few. However, they cannot be directly used to implement unidirectional light transport and its manipulation [8,9], an essential and more difficult task than others in all-optical networks. In this respect, only in recent years significant progress has been made by considering moving photonic crystals of driven atoms $[10,11]$ and optical materials with parity-time $(P T)$ symmetry $[12,13]$. As compared to traditional photonic crystals, $P T$-symmetric materials are periodically modulated not only in terms of the real part $n^{\prime}$ but also the imaginary part $n^{\prime \prime}$ of the refractive index $n$, exhibiting a delicate balance of gain and loss alternately along the modulation direction.

On the other hand, non-Hermitian Hamiltonians could exhibit real eigenvalues if they are invariant under the combination of parity and time-reversal operations [14]. Hinging on the mathematical isomorphism between the Schrodinger equation and the Maxwell paraxial wave equation, this unusual behavior has been implemented to realize fairly interesting optical settings in media whose refractive indices satisfy $n(z)=n^{*}(-z)$ [15-17]. Such an even optical $P T$ symmetry has been used, in fact, in the realization of coherent perfect absorbers [18-20], giant wave amplification [21,22], spatial optical switching [23], quantum state transformation [24], and quantum state discrimination [25]. So far most even $P T$-symmetric materials are solid-state systems that consist of periodically arranged microstructures bearing on the whole balanced gain and loss. Homogeneous vapors of multilevel ultracold atoms can further be used to attain even PT- symmetric optical potentials though via rather complicated spatial modulations of the driving fields [26-28]. These atomic proposals have the obvious advantages of real-time all-optical tunable and reconfigurable capabilities. Very recently, the odd optical PT-symmetry with $n(z)=-n^{*}(-z)$ was also considered [29], which however requires a more involved and even impractical balance of positive and negative real refractive indices.

In contrast, media with $P T$-antisymmetric susceptibilities, i.e., $\chi(z)=-\chi^{*}(-z)$, requiring neither optical gain nor negative refraction is explored here. The present work builds on a recent proposal [30] where such a medium could be realized in a one-dimensional (1D) atomic lattice and extends it by presenting a detailed analysis on the connection between the unidirectional reflectionless properties of such lattices and the non-Hermitian $(\mathrm{NH})$ degeneracies of their scattering matrix. In particular, we present a thorough discussion of the singular behavior of the scattering matrix eigenvalues that are, in turn, contrasted to those of the corresponding scattering matrices for media with $P T$-even and $P T$-odd refractive indices.

Driven atomic lattices have aroused great interest of research for achieving controlled photonic band gaps, radiation damping enhancement, and two-color lasing oscillation [31-34]. Here we assume that all trapped atoms-the density modulation of which is dominated by a cosine term-are driven into the four-level $N$ configuration with a far-detuned dressing field applied to induce the dynamic shift of one empty ground level. We find that a probe field may experience the $P T$-antisymmetric susceptibility when this dressing field has a traveling-wave (TW) and a standing-wave (SW) component such that the dynamic frequency shift is modulated as a sine function along the lattice direction. That is, real and imaginary parts of the probe susceptibility are, respectively, an odd and an even function of the lattice position in each dipole trap. This dressing field modulation destroys the two-photon resonance condition between the probe and coupling fields in the regime of electromagnetically induced transparency (EIT) [35] and therefore we consider indeed passive atomic lattices with 
essential absorptive loss. Such optical $P T$ antisymmetry then allows us to observe high-contrast unbalanced reflectivities and even fully unidirectional reflection with finite transmission at one NH-degeneracy point. It is of special interest that (i) a vanishing probe reflectivity at the probe resonance can be easily switched between the two lattice ends by changing the detuning signs of both TW and SW dressing components; (ii) fully unidirectional reflection can be attained even out of the probe resonance when the dynamic level shift deviates from a sine function of the lattice position. Last but not least, both $P T$-antisymmetric susceptibility and unidirectional light reflection depend also critically on the dynamically controlled Gaussian density distribution in each dipole trap. A coupled-mode analysis is also done to illustrate how fully unidirectional reflection requires that $\chi^{\prime}(z)$ and $\chi^{\prime \prime}(z)$ be exactly mismatched by a half-period to display an odd and an even function, respectively, in the 1D spatial modulation.

\section{PT -ANTISYMMETRIC SUSCEPTIBILITY}

We consider here an ensemble of cold atoms distributed into the optical lattices of dipole traps formed by the retroreflecting red-detuned laser beams of wavelength $\lambda_{o}$ [Fig. 1(a)]. The resultant atomic lattices have a period $a=0.5 \lambda_{o} / \cos \theta_{o}$ with $\theta_{o}$ being a small angle between the dipole-trap laser beams and the lattice axis along $\vec{z}$. These trapped atoms exhibit one typical Gaussian density distribution $N_{j}(z)=N_{0} \exp \left[-\left(z-z_{j}\right)^{2} / d^{2}\right]$ in each period with $d$ being the $1 / e$ half-width and $z_{j}$ the $j$ th lattice center. Such periodically distributed atoms are driven into the four-level $N$ configuration by three coherent fields of frequencies (amplitudes) $\omega_{p}\left(\mathbf{E}_{p}\right), \omega_{c}\left(\mathbf{E}_{c}\right)$, and $\omega_{d 1}\left(\mathbf{E}_{d 1}\right)$ or $\omega_{d 2}\left(\mathbf{E}_{d 2}\right)$ [Fig. 1(b)]. The weak probe field $\omega_{p}$, moderate coupling field $\omega_{c}$, and strong dressing field $\omega_{d 1}$ or $\omega_{d 2}$ interact, respectively, with the dipole-allowed transitions $|1\rangle \leftrightarrow|3\rangle$, $|2\rangle \leftrightarrow|3\rangle$, and $|2\rangle \leftrightarrow|4\rangle$. Corresponding frequency detunings (Rabi frequencies) are $\Delta_{p}=\omega_{p}-\omega_{31}, \Delta_{c}=\omega_{c}-\omega_{32}$, and $\Delta_{d 1}=\omega_{d 1}-\omega_{42}$ or $\Delta_{d 2}=\omega_{d 2}-\omega_{42}\left(\Omega_{p}=\mathbf{E}_{p} \mathbf{d}_{13} / 2 \hbar\right.$, $\Omega_{c}=\mathbf{E}_{c} \mathbf{d}_{23} / 2 \hbar$, and $\Omega_{d 1}=\mathbf{E}_{d 1} \mathbf{d}_{24} / 2 \hbar$ or $\left.\Omega_{d 2}=\mathbf{E}_{d 2} \mathbf{d}_{24} / 2 \hbar\right)$ defined as usual with $\omega_{i j}$ being resonant transition frequencies and $\mathbf{d}_{i j}$ being electric-dipole moments.

In what follows, we assume that $\Omega_{d 1}=\Omega_{d}, \Omega_{d 2}=$ $\sqrt{2} \Omega_{d} \cos \left(k_{d} z-\phi_{d}\right)$, and $\Delta_{d 1}=-\Delta_{d 2}=\Delta_{d}$ with the restriction $\left|\Delta_{d}\right| \gg \Omega_{d}$. In this case, the $|2\rangle \leftrightarrow|4\rangle$ transition is driven by a TW dressing component and a SW dressing component. The two transition pathways contributed by either a TW or a SW dressing component don't interfere with each other due to a giant frequency difference $2\left|\Delta_{d}\right|$. Under the rotating-wave and electric-dipole approximations, we can write down the interaction Hamiltonian and then obtain the density matrix equations

$$
\begin{aligned}
& \partial_{t} \rho_{12}=-\left(\gamma_{12}+i \Delta_{12}\right) \rho_{12}-i \Omega_{c} \rho_{13}-i \Omega_{d 1} \rho_{14}^{(1)}-i \Omega_{d 2} \rho_{14}^{(2)}, \\
& \partial_{t} \rho_{13}=-\left(\gamma_{13}+i \Delta_{p}\right) \rho_{13}-i \Omega_{c}^{*} \rho_{12}-i \Omega_{p}^{*},
\end{aligned}
$$

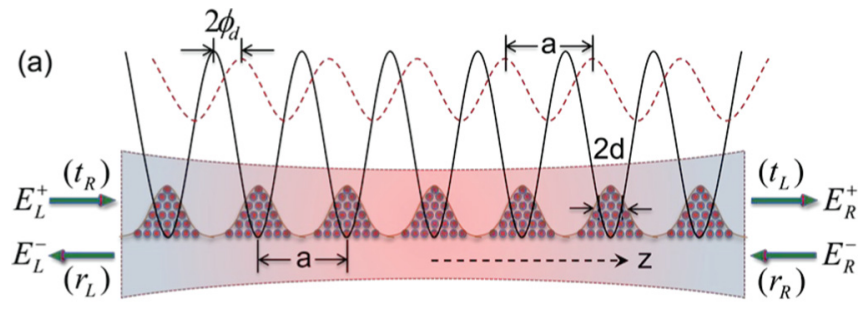

(b)

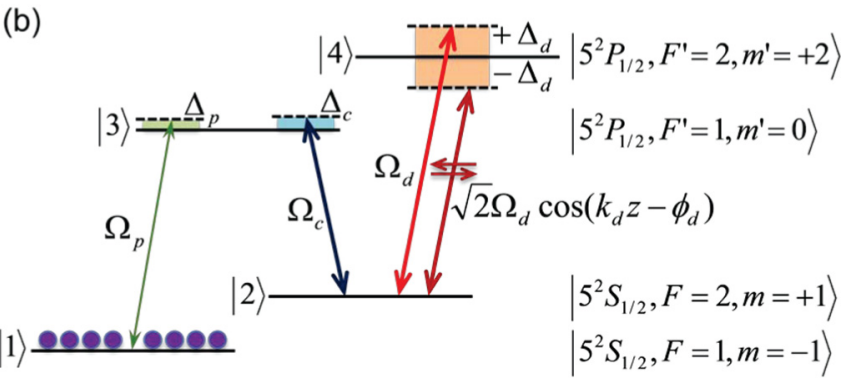

FIG. 1. (Color online) (a) Typical scattering experiment geometry where a pair of incident electric-field amplitudes $\left(E_{\mathrm{L}}^{+}, E_{\mathrm{R}}^{-}\right)$are scattered by a periodic lattice of atoms into outgoing electric-field amplitudes $\left(E_{\mathrm{L}}^{-}, E_{\mathrm{R}}^{+}\right)$. These four amplitudes are connected by the transformation $S$ in Eq. (11). For fields $\left(E_{\mathrm{L}}^{+}\right)$incident from the left, e.g., outgoing amplitudes consist of waves $\left(E_{\mathrm{R}}^{+}\right)$transmitted with amplitude $t_{\mathrm{L}}$ in the $+z$ direction as well as waves $\left(E_{\mathrm{L}}^{-}\right)$reflected with amplitude $r_{\mathrm{L}}$ in the $-z$ direction. Similarly for fields $\left(E_{\mathrm{R}}^{-}\right)$incident from the right with transmission and reflection amplitudes $t_{\mathrm{R}}$ and $r_{\mathrm{R}}$. This atomic periodic structure arises from a $1 \mathrm{D}$ optical lattice (black solid) of period $a$ where ultracold ${ }^{87} \mathrm{Rb}$ atoms are trapped at the bottom of dipole traps with a Gaussian distribution of full width $2 d$. Each atom experiences also a dynamic level shift (red dashed) with a spatial period $a$ and a phase shift $2 \phi_{d}$ relative to the corresponding dipole trap. (b) All atoms are driven into the four-level $N$ configuration by a weak near-resonant probe field ( green) on the $|1\rangle \leftrightarrow|3\rangle$ transition, a moderate near-resonant coupling field (blue) on the $|2\rangle \leftrightarrow|3\rangle$ transition, and a strong far-detuned dressing field (red) on the $|2\rangle \leftrightarrow|4\rangle$ transition. Both probe $\left(\Omega_{p}, \Delta_{p}\right)$ and coupling $\left(\Omega_{c}, \Delta_{c}\right)$ fields are assumed to travel in the $z$ direction. The dressing field has instead a TW component $\left(\Omega_{d},+\Delta_{d}\right)$ traveling in the $x$ direction and a SW component $\left[\sqrt{2} \Omega_{d} \cos \left(k_{d} z-\phi_{d}\right),-\Delta_{d}\right]$ modulated in the $z$ direction. The empty ground level $|2\rangle$ then exhibits a dynamic shift $\delta_{d s}(z)=\delta_{d 0} \cos \left[2\left(k_{d} z-\phi_{d}\right)\right]$ with $\delta_{d 0}=\Omega_{d}^{2} / \Delta_{d}$ and $k_{d}=\pi / a$ in the limit of $\left|\Delta_{d}\right| \gg \Omega_{d}$ and $\Omega_{d} \gg \gamma_{14}$ (see the Appendix).

$$
\begin{aligned}
& \partial_{t} \rho_{14}^{(1)}=-\left(\gamma_{14}+i \Delta_{14}^{(1)}\right) \rho_{14}^{(1)}-i \Omega_{d 1}^{*} \rho_{12}, \\
& \partial_{t} \rho_{14}^{(2)}=-\left(\gamma_{14}+i \Delta_{14}^{(2)}\right) \rho_{14}^{(2)}-i \Omega_{d 2}^{*} \rho_{12},
\end{aligned}
$$

with $\rho_{11} \simeq 1$ and $\rho_{22} \simeq \rho_{33} \simeq \rho_{44} \simeq 0$ in the limit of a weak probe field. In Eqs. (1), we have phenomenally introduced the coherence dephasing rates $\gamma_{12}, \gamma_{13}$, and $\gamma_{14}$ as usual and simultaneously defined the multiphoton detunings $\Delta_{12}=\Delta_{p}-\Delta_{c}$, $\Delta_{14}^{(1)}=\Delta_{12}+\Delta_{d}$, and $\Delta_{14}^{(2)}=\Delta_{12}-\Delta_{d}$ for convenience. Setting $\partial_{t} \rho_{i j}=0$ in Eqs. (1), we attain the steady-state solution

$$
\rho_{31}(z)=\frac{i \Omega_{p}\left[\left(\gamma_{12}-i \Delta_{12}\right)+\Omega_{d}^{2} /\left(\gamma_{14}-i \Delta_{12}-i \Delta_{d}\right)+2 \Omega_{d}^{2} \cos ^{2}\left(k_{d} z-\phi_{d}\right) /\left(\gamma_{14}-i \Delta_{12}+i \Delta_{d}\right)\right]}{\Omega_{c}^{2}+\left(\gamma_{13}-i \Delta_{p}\right)\left[\left(\gamma_{12}-i \Delta_{12}\right)+\Omega_{d}^{2} /\left(\gamma_{14}-i \Delta_{12}-i \Delta_{d}\right)+2 \Omega_{d}^{2} \cos ^{2}\left(k_{d} z-\phi_{d}\right) /\left(\gamma_{14}-i \Delta_{12}+i \Delta_{d}\right)\right]} .
$$



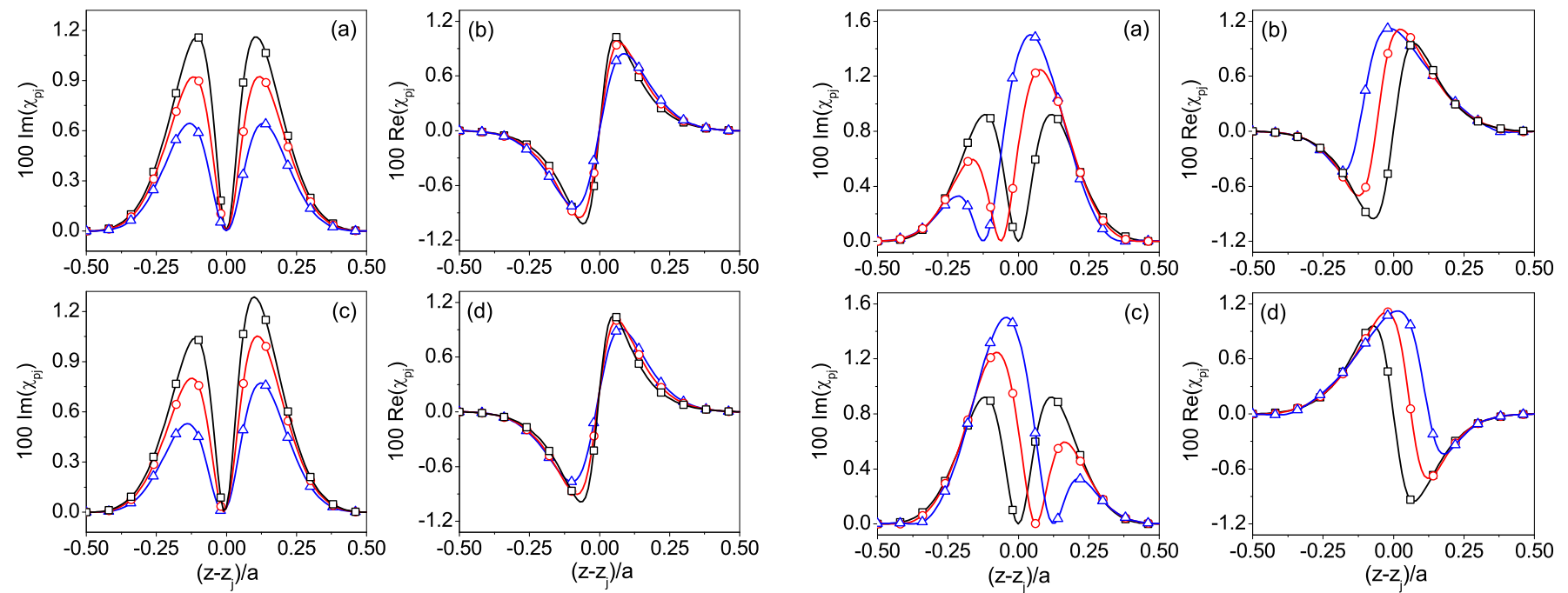

FIG. 2. (Color online) Plots of $\operatorname{Im}\left(\chi_{p j}\right)(\mathrm{a}, \mathrm{c})$ and $\operatorname{Re}\left(\chi_{p j}\right)(\mathrm{b}, \mathrm{d})$ vs lattice position $z-z_{j}$ in the $j$ th period with $\gamma_{12}=2.0 \mathrm{kHz}, \gamma_{13}=$ $\gamma_{14}=3.0 \mathrm{MHz}, \Delta_{c}=0, \Omega_{c}=2.5 \mathrm{MHz}, \Delta_{d}=300 \mathrm{MHz}, \phi_{d}=$ $\pi / 4, a=5 d \simeq 400 \mathrm{~nm}, d_{13}=2.0 \times 10^{-29} \mathrm{Cm}$, and $N_{0}=2.0 \times$ $10^{12} \mathrm{~cm}^{-3}$. Panels $(\mathrm{a}, \mathrm{b})$ refer to $\Delta_{p}=0$, while panels $(\mathrm{c}, \mathrm{d})$ refer to $\Delta_{p}=0.2 \mathrm{MHz}$. The black-square, red-circle, and blue-triangle curves are obtained, respectively, with $\Omega_{d}=27.4 \mathrm{MHz}, 32.9 \mathrm{MHz}$, and $38.3 \mathrm{MHz}$.

For simplicity, we further assume $\gamma_{13}=\gamma_{14}=\gamma, \gamma \gg \gamma_{12}$, and $\Delta_{c}=0$, while focusing on the special case of $\left|\Delta_{d}\right| \gg \Omega_{d}$, $\Omega_{d} \gg \gamma$, and $\gamma \gtrsim \Delta_{p}$. These conditions allow us to rewrite Eq. (2) into a much simpler form

$$
\rho_{31}(z) \simeq \frac{i \Omega_{p}\left\{\gamma_{12}-i\left[\Delta_{p}+\delta_{d s}(z)\right]\right\}}{\Omega_{c}^{2}+\left(\gamma-i \Delta_{p}\right)\left\{\gamma_{12}-i\left[\Delta_{p}+\delta_{d s}(z)\right]\right\}},
$$

with a dynamic shift $\delta_{d s}(z)=\delta_{d 0} \cos \left[2\left(k_{d} z-\phi_{d}\right)\right]$ of maximal amplitude $\delta_{d 0}=\Omega_{d}^{2} / \Delta_{d}$ for the ground level $|2\rangle$. Considering the Gaussian density distribution $N_{j}(z)$, it is straightforward to attain the twofold modulated probe susceptibility in the $j$ th lattice period

$$
\chi_{p_{j}}(z)=\frac{i \alpha_{j}(z)\left\{\gamma_{12}-i\left[\Delta_{p}+\delta_{d s}(z)\right]\right\}}{\Omega_{c}^{2}+\left(\gamma-i \Delta_{p}\right)\left\{\gamma_{12}-i\left[\Delta_{p}+\delta_{d s}(z)\right]\right\}},
$$

with $\alpha_{j}(z)=N_{j}(z) d_{13}^{2} / 2 \varepsilon_{0} \hbar$. Notice that both forward and backward beams of the SW dressing component may have a small angle $\theta_{d}$ relative to the lattice axis $\vec{z}$ with $k_{d}=$ $2 \pi \cos \left(\theta_{d}\right) / \lambda_{d}$ and we can attain $k_{d}=\pi / a$ by accurately modulating the two small angles $\theta_{o}$ and $\theta_{d}$ even if $\lambda_{d} \neq \lambda_{o}$. In addition, it is worth stressing that $\delta_{d s}(z)$ turns out to be a sine function of the lattice position $z$ with $\phi_{d}= \pm \pi / 4$. The correlated Gaussian-sine modulation is then expected to yield an antisymmetric probe susceptibility with its real (imaginary) part being an odd (even) function of the lattice position. Further technical details related to the dynamic control of the probe susceptibility modulation in the four-level $N$ scheme here proposed are discussed in the Appendix.

In Fig. 2, we plot imaginary and real parts of probe susceptibility $\chi_{p j}(z)$ as a function of lattice position $z$ with $\Delta_{p}=0$ in panels (a) and (b) but $\Delta_{p}=0.2 \mathrm{MHz}$ in panels (c) and (d). It is clear that $\operatorname{Im}\left[\chi_{p j}(z)\right]$ and $\operatorname{Re}\left[\chi_{p j}(z)\right]$ are spatially

FIG. 3. (Color online) Plots of $\operatorname{Im}\left(\chi_{p j}\right)(\mathrm{a}, \mathrm{c})$ and $\operatorname{Re}\left(\chi_{p j}\right)(\mathrm{b}, \mathrm{d})$ vs lattice position $z-z_{j}$ in the $j$ th period with the same parameters as in Fig. 2 except $\Delta_{p}=0, \Omega_{d}=32.9 \mathrm{MHz}$, and $\phi_{d}=+\pi / 4$ (black square), $+3 \pi / 16$ (red circle), and $+\pi / 8$ (blue triangle) in panels (a,b); $\phi_{d}=-\pi / 4$ (black square), $-3 \pi / 16$ (red circle), and $-\pi / 8$ (blue triangle) in panels (c, d).

modulated in distinct ways along the lattice axis for a given probe detuning $\Delta_{p}$. At probe resonance $\Delta_{p}=0$, in particular, $\operatorname{Im}\left[\chi_{p j}(z)\right]$ and $\operatorname{Re}\left[\chi_{p j}(z)\right]$ are respectively an exact even and odd function of the lattice position relative to the lattice center, indicating that our atomic lattices exhibit perfect $P T$ antisymmetry characterized by $\chi_{p j}(+z)=-\chi_{p j}^{*}(-z)$. With $\Delta_{p}=0.2 \mathrm{MHz}$, however, $\operatorname{Re}\left[\chi_{p j}(+z)+\chi_{p j}(-z)\right]$ deviates slightly while $\operatorname{Im}\left[\chi_{p j}(+z)-\chi_{p j}(-z)\right]$ deviates severely from zero to result in considerable errors in the $P T$ antisymmetry. In addition, it is convenient to manipulate the ratio between maximum amplitudes of $\operatorname{Im}\left[\chi_{p j}(+z)\right]$ and $\operatorname{Re}\left[\chi_{p j}(+z)\right]$ by changing the dressing Rabi frequency $\Omega_{d}$ (i.e., the dynamic frequency shift $\delta_{d 0}$ ). This straightforward manipulation opportunity is significant for potential applications, e.g., in achieving high-contrast asymmetric reflectivities and even fully unidirectional reflection.

In Fig. 3, we examine how the relative phase $\phi_{d}$ between the SW dressing field component and the atomic density modulation affects the $P T$ antisymmetry of the probe susceptibility $\chi_{p j}(z)$. We find that the perfect $P T$ antisymmetry is attained only for $\phi_{d}= \pm(1 / 4,3 / 4) \pi$, promising an exact sine modulation of the dynamic level shift $\delta_{d s}$. With the decreasing [panels (a) and (b)] or increasing [panels (c) and (d)] of $\phi_{d}$, however, the minima of both probe absorption [panels (a) and (c)] and dispersion [panels (b) and (d)] simultaneously move left or right to result in worse and worse $P T$ antisymmetry. As the relative phase is $\phi_{d}=(0, \pm 1 / 2) \pi$, the $P T$ antisymmetry of probe susceptibility will be completely destroyed in the presence of an exact cosine modulation of the dynamic level shift $\delta_{d s}$. That is, both real and imaginary parts of the probe susceptibility $\chi_{p j}(z)$ are modulated into an even function of the lattice position (not shown).

The probe susceptibility in Eq. (4) has a lot of spatial Fourier components oscillating at $\exp \left[ \pm 2 i m k_{d} z\right]$ with 

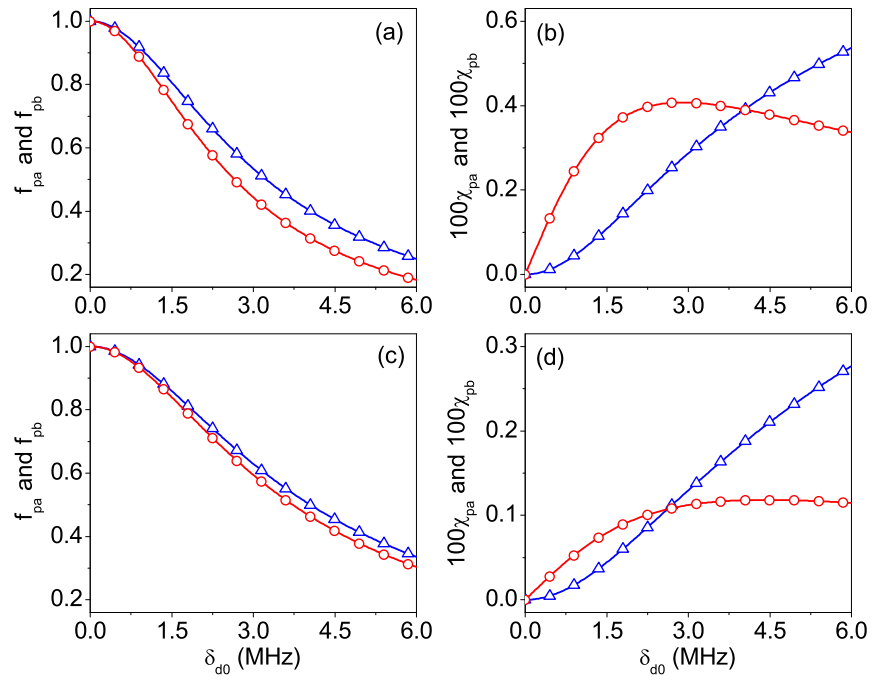

FIG. 4. (Color online) Plots of $f_{p a}$ and $f_{p b}(\mathrm{a}, \mathrm{c}) ; \chi_{p a}$ and $\chi_{p b}$ (b, d) vs maximal dynamic shift $\delta_{d 0}$ with the same parameters as in Fig. 2 except $\Delta_{p}=0$ and $a=5 d=400 \mathrm{~nm}(\mathrm{a}, \mathrm{b}) ; a=10 d=$ $400 \mathrm{~nm}(\mathrm{c}, \mathrm{d})$. The red-circle and blue-triangle curves correspond, respectively, to $f_{p a}$ and $f_{p b}$ in panels $(\mathrm{a}, \mathrm{c})$ and to $\chi_{p a}$ and $\chi_{p b}$ in panels $(b, d)$.

$m \in\{0,1,2, \ldots\}$, but only its zero- and first-order components are responsible for the dynamic propagation of a probe field with $k_{p} \simeq \pm k_{d}$. It is rather involved or even impossible to attain analytical expressions of the zero- and first-order susceptibility components due to the correlated Gaussian and sine modulations. Therefore, we choose fully numerical Fourier calculations in the case of $\Delta_{p}=0, \phi_{d}=\pi / 4$, and $\gamma_{12} \rightarrow 0$ to examine the zero- and first-order susceptibility components with

$$
\begin{aligned}
\chi_{p j}(z)= & \frac{-\alpha_{0} \delta_{d 0} \sin \left(2 k_{d} z\right)}{\Omega_{c}^{2}+i \gamma \delta_{d 0} \sin \left(2 k_{d} z\right)} \exp \left[-\left(z-z_{j}\right)^{2} / d^{2}\right] \\
= & \frac{\alpha_{0} x}{\gamma} \frac{-\sin \left(2 k_{d} z\right)}{1+i x \sin \left(2 k_{d} z\right)} \exp \left[-\left(z-z_{j}\right)^{2} / d^{2}\right] \\
= & i \chi_{p 0}+i \chi_{p a} \cos \left(2 k_{d} z\right)-\chi_{p b} \sin \left(2 k_{d} z\right)+\cdots \\
= & \frac{\alpha_{0} x}{\gamma}\left\{f_{p b}\left(b_{0}-b_{2}\right)\left[i x-\sin \left(2 k_{d} z\right)\right]\right. \\
& \left.+\frac{i x}{2} f_{p a}\left(b_{1}-b_{3}\right) \cos \left(2 k_{d} z\right)\right\}+\cdots
\end{aligned}
$$

in which for simplicity we have set $x=\gamma \delta_{d 0} / \Omega_{c}^{2}$, while $b_{n}=\sqrt{\pi}(d / a) \exp \left[-(n \pi d / a)^{2}\right]$ is attained via the Fourier transform on a Gaussian density distribution.

In Fig. 4, we plot $f_{p a}, f_{p b}, \chi_{p a}$, and $\chi_{p b}$ of first-order susceptibility components as a function of maximal dynamic shift $\delta_{d 0}$ with Gaussian widths $d=a / 5$ in panels (a) and (b) but $d=a / 10$ in panels (c) and (d). The two left panels show that $f_{p a}$ and $f_{p b}$ become smaller and smaller with the increasing of $\delta_{d 0}$ but remain rather close in the synchronous attenuation (especially true for a smaller $d$ ) independent of $\delta_{d 0}$. This means that rather accurate results could be attained even if we set $f_{p a}=f_{p b}=1.0$ (available in the limit of $x \rightarrow 0$ ) in Eq. $(5 \mathrm{c})$ to predict an exceptional point of $\delta_{d 0}$ corresponding to $\chi_{p a}=$ $\chi_{p b}$. In fact, the exceptional point is located at $\delta_{d 0} \simeq 2.62 \mathrm{MHz}$ with $d=a / 10$ in panel (d) but will move slightly to $\delta_{d 0} \simeq$ $2.75 \mathrm{MHz}$ if we assume $f_{p a}=f_{p b}=1.0$ (examined but not shown). Comparing panel (d) to panel (b), we find that the exceptional point occurs at a larger maximal dynamic shift $\delta_{d 0} \simeq 4.03 \mathrm{MHz}$ in the presence of a larger Gaussian width $d=a / 5$. So a narrower Gaussian distribution is preferred for achieving the exceptional point of $\chi_{p a}=\chi_{p b}$ with a smaller maximal dynamic shift $\delta_{d 0}$ (dressing Rabi frequency $\Omega_{d}$ ). The controlled Gaussian width $d$ depends on the trapping depth $U_{0}$ of dipole potentials and the average temperature $T$ of trapped atoms via $d=\left(\lambda_{o} \sqrt{\kappa_{B} T}\right) /\left(2 \pi \sqrt{2 U_{0}}\right)$ [32].

\section{FULLY UNIDIRECTIONAL REFLECTION}

One important application of $P T$-antisymmetric probe susceptibilities is to realize high-contrast asymmetric reflectivities and even fully unidirectional reflection. This can be examined either by incorporating the accurate Eq. (4) into transfer-matrix equations or by incorporating the approximate Eq. (5b) into coupled-mode equations. The former method allows exact full numerical solutions with arbitrary probe detunings $\Delta_{p} \neq 0$, while the latter method is favorable for getting physical insights with a vanishing probe detuning $\Delta_{p}=0$.

We start with the more general case $\Delta_{p} \neq 0$ to derive the $2 \times 2$ unimodular transfer matrix $M_{j}$ of a single lattice period. To this end, we divide each period into, e.g., 100 layers, of identical thickness $\delta z$ but distinct density $N_{j}\left(z_{l}\right)$ for $l \in\{1,100\}$ and write down the primary transfer matrix of each ultrathin layer

$$
m_{j}\left(z_{l}\right)=\frac{1}{t_{j}\left(z_{l}\right)}\left[\begin{array}{cc}
t_{j}^{2}\left(z_{l}\right)-r_{j}^{2}\left(z_{l}\right) & +r_{j}\left(z_{l}\right) \\
-r_{j}\left(z_{l}\right) & 1
\end{array}\right],
$$

where the elementary reflection and transmission coefficients, $r_{j}\left(z_{l}\right)$ and $t_{j}\left(z_{l}\right)$, are determined by the complex refractive index $n_{p j}\left(z_{l}\right) \simeq 1+\chi_{p_{j}}\left(z_{l}\right) / 2$ based on Eq. (4) [36]. With $M_{j}=m_{j}\left(z_{1}\right) \cdots m_{j}\left(z_{l}\right) \cdots m_{j}\left(z_{100}\right)$, we attain the ultimate transfer matrix $M_{0}^{2 k+1}=M_{-k} \cdots M_{0} \cdots M_{+k}$ for finite atomic lattices of realistic length $L=(2 k+1) a$. Then it is straightforward to compute probe transmissivities and reflectivities on both lattice ends

$$
\begin{aligned}
& T_{L}=T_{R}=|t|^{2}=\left|\frac{1}{M_{0(22)}^{2 k+1}}\right|^{2}, \\
& R_{L}=\left|r_{L}\right|^{2}=\left|\frac{M_{0(12)}^{2 k+1}}{M_{0(22)}^{2 k+1}}\right|^{2}, \quad R_{R}=\left|r_{R}\right|^{2}=\left|\frac{M_{0(21)}^{2 k+1}}{M_{0(22)}^{2 k+1}}\right|^{2}
\end{aligned}
$$

using relevant matrix elements of $M_{0}^{2 k+1}$.

In Fig. 5, we plot probe reflectivities and transmissivities as a function of maximal dynamic shift $\delta_{d 0}$ for three probe detunings. In the left panels with $d=a / 5$, we find that $R_{L}$ is very different from $R_{R}$ and $T$ is completely vanishing when $\left|\delta_{d 0}\right|$ is not too small. In the right panels with $d=a / 10$, we find that a larger $\left|\delta_{d 0}\right|$ is required to have a completely vanishing $T$ but a clear difference always exists between $R_{L}$ and $R_{R}$. We have, in particular, $R_{L} \rightarrow 0$ and $T \rightarrow 0$ but $R_{R} \simeq 0.26$ near an exceptional point at $\delta_{d 0} \simeq 4.0 \mathrm{MHz}$ in the left panels; $R_{L} \rightarrow 0$ and $T \rightarrow 0.04$ but $R_{R} \simeq 0.58$ near an exceptional point at 

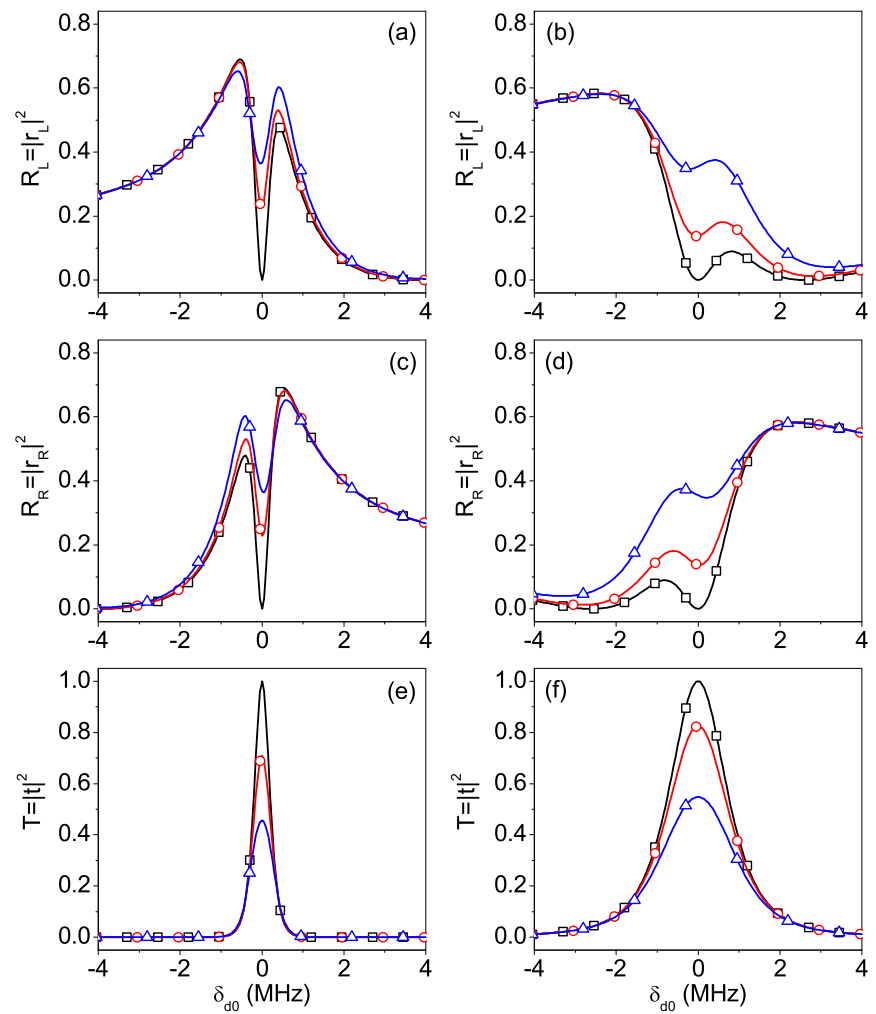

FIG. 5. (Color online) Plots of $R_{L}$ (a, b), $R_{R}$ (c, d), and $T$ (e,f) vs maximal dynamic shift $\delta_{d 0}$ for $\Delta_{p}=0.0 \mathrm{MHz}$ (black square), $0.1 \mathrm{MHz}$ (red circle), and $0.2 \mathrm{MHz}$ (blue triangle). Here we consider a finite lattice of length $L=0.6 \mathrm{~mm}$ with $a=5 d=400 \mathrm{~nm}$ (left panels) and $a=10 d=400 \mathrm{~nm}$ (right panels). Other parameters are the same as in Fig. 2.

$\delta_{d 0} \simeq 2.6 \mathrm{MHz}$ in the right panels. The exceptional point shift from $\delta_{d 0} \simeq 4.0 \mathrm{MHz}$ with $d=a / 5$ to $\delta_{d 0} \simeq 2.6 \mathrm{MHz}$ with $d=a / 10$ is easily understood with the predictions in Fig. 4, while the larger nonvanishing reflectivity $R_{R}$ at the exceptional point for the sharper Gaussian distribution $d$ is a new result. In addition, a nonvanishing probe detuning may evidently reduce the reflectivity contrast

$$
C_{R}=\left(R_{R}-R_{L}\right) /\left(R_{R}+R_{L}\right)
$$

even at the exceptional point, and it is convenient to swap the values of reflectivities $R_{L}$ and $R_{R}$ by changing the sign of dynamic shift $\delta_{d 0}$. Then the $P T$-antisymmetric susceptibility entails strongly unbalanced probe reflectivities in the presence of significant loss, making our atomic lattices visible upon (light) reflection only from one side at the exceptional point.

In Fig. 6, we further examine how the relative phase $\phi_{d}$ of dressing field components affects reflectivity $R_{L}$, reflectivity $R_{R}$, transmissivity $T$, and contrast $C_{R}$ at the exceptional point. It is clear that we have the balanced reflectivities $R_{L}=R_{R}$ only at $\phi_{d}= \pm \pi / 2$ with a given probe detuning, while $R_{L}$ and $R_{R}$ change in the opposite ways when $\phi_{d}$ is increased or decreased within the range of $\{-\pi / 2,+\pi / 2\}$. In addition, we can attain fully unidirectional reflection even with a nonzero probe detuning as long as $\phi_{d}$ is modulated to be slightly different from $\pm \pi / 4$. In fact, the maximal reflectivity contrast $C_{R} \rightarrow \pm 1$ is attained at $\phi_{d}= \pm 0.25 \pi$
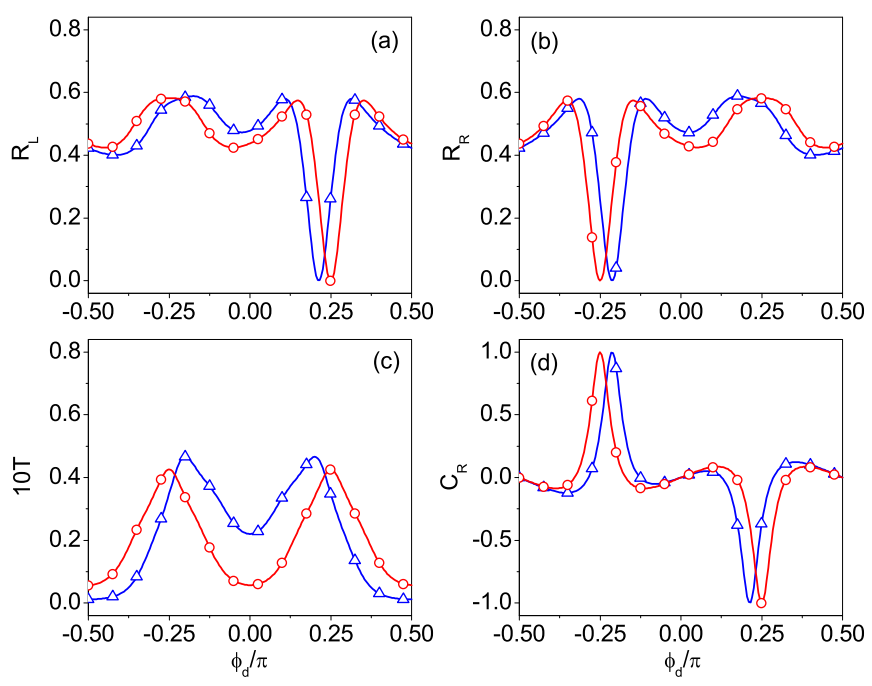

FIG. 6. (Color online) Plots of $R_{L}$ (a), $R_{R}$ (b), $T$ (c), and $C_{R}$ (d) vs relative phase $\phi_{d}$ for $\Delta_{p}=0.0 \mathrm{MHz}$ (red circle) and $0.5 \mathrm{MHz}$ (blue triangle). Other parameters are the same as in Fig. 5 except $\delta_{d 0}=2.6 \mathrm{MHz}$ and $a=10 d=400 \mathrm{~nm}$.

with $\Delta_{p}=0$ but at $\phi_{d} \simeq \pm 0.21 \pi$ with $\Delta_{p}=0.5 \mathrm{MHz}$. So the exact sine modulation corresponding to $\phi_{d}= \pm \pi / 4$ is not strictly necessary to realize fully unidirectional reflection characterized by $C_{R} \rightarrow \pm 1$, as discussed at the end of Sec. IV in terms of a NH-degeneracy line in the $\left(\Delta_{p}, \phi_{d}\right)$ plane. In any case, the reflectivity contrast $C_{R}$ is sensitive to probe detuning $\Delta_{p}$ and we estimate that light signals of full widths $\gtrsim 10 \mu \mathrm{s}$ should be adopted in potential applications to avoid significant pulse deformation.

Now we consider a more specific case $\Delta_{p}=0$ to establish the coupled-mode equations in the limit of $\gamma_{12} \rightarrow 0$ and $\phi_{d}=$ $\pi / 4$. We stress once again that the neglected higher-order components oscillating at $\exp \left[ \pm 2 i m k_{d} z\right]$ with $m \in\{2,3,4, \ldots\}$ will not contribute to the propagation dynamics of a probe field decomposed into, e.g., $E_{p}(z)=E_{f}(z) \exp \left[+i k_{d} z\right]+$ $E_{b}(z) \exp \left[-i k_{d} z\right]$ due to Bragg scattering. Substituting the zero- and first-order components of $\chi_{p j}(z)$ in Eq. (5b) into the 1D Helmholtz equation $\partial_{z}^{2} E_{p}(z)+k_{p}^{2} n_{p}^{2}(z) E_{p}(z)=0$, keeping only the synchronous terms and eliminating the second-order smaller terms, it is then straightforward to obtain the following two coupled-mode equations:

$$
\begin{aligned}
\partial_{z} E_{f} & =-k_{d} \chi_{p 0} E_{f} / 4-k_{d}\left[\chi_{p b}+\chi_{p a}\right] E_{b} / 4 \\
& =-\eta_{1}\left[x E_{f}+\left(1+x \eta_{2} / 2\right) E_{b}\right], \\
\partial_{z} E_{b} & =+k_{d} \chi_{p 0} E_{b} / 4-k_{d}\left[\chi_{p b}-\chi_{p a}\right] E_{f} / 4 \\
& =+\eta_{1}\left[x E_{b}-\left(1-x \eta_{2} / 2\right) E_{f}\right],
\end{aligned}
$$

with $\quad k_{p} \simeq k_{d}, \quad \chi_{p 0}=x_{p b}, \quad \eta_{1}=k_{d} \chi_{p b} / 4, \quad$ and $\quad \eta_{2}=$ $(2 / x)\left(\chi_{p a} / \chi_{p b}\right)$. It is easy to see from Eqs. (9) that the forward $\left(E_{f}\right)$ and backward $\left(E_{b}\right)$ field components will experience different nonlinear feedback if we have $x \eta_{2} \neq 0\left(\chi_{p a} \neq 0\right)$; $x \eta_{2}= \pm 2$ correspond to the exceptional points of optical $P T$ antisymmetry where one field component suffers only itself linear absorption, i.e., loses all nonlinear feedback from the other field component. 


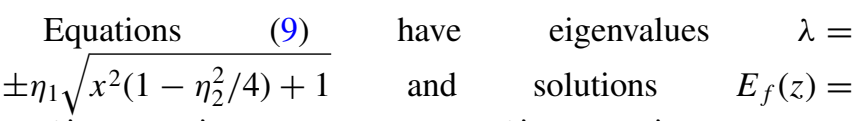
$c_{1} e^{+\lambda z}+c_{2} e^{-\lambda z}$ and $E_{b}(z)=d_{1} e^{+\lambda z}+d_{2} e^{-\lambda z}$. With the boundary conditions $E_{f}(0)=E_{L 0}$ and $E_{b}(L)=0$ as well as $E_{f}(0)=0$ and $E_{b}(L)=E_{R 0}$, we can determine from Eqs. (9) the four unknown coefficients $\left(c_{1,2}\right.$ and $\left.d_{1,2}\right)$ and thus left-hand and right-hand transmissivities and reflectivities

$$
\begin{aligned}
T & =\left|\frac{2 \lambda}{\left(\lambda+x \eta_{1}\right) e^{+\lambda L}+\left(\lambda-x \eta_{1}\right) e^{-\lambda L}}\right|^{2}, \\
R_{L} & =\left|\frac{+\eta_{1}\left(1-x \eta_{2} / 2\right)\left(e^{+\lambda L}-e^{-\lambda L}\right)}{\left(\lambda+x \eta_{1}\right) e^{+\lambda L}+\left(\lambda-x \eta_{1}\right) e^{-\lambda L}}\right|^{2}, \\
R_{R} & =\left|\frac{-\eta_{1}\left(1+x \eta_{2} / 2\right)\left(e^{+\lambda L}-e^{-\lambda L}\right)}{\left(\lambda+x \eta_{1}\right) e^{+\lambda L}+\left(\lambda-x \eta_{1}\right) e^{-\lambda L}}\right|^{2},
\end{aligned}
$$

which shows that $T_{L} \equiv T_{R}=T$ but $R_{L}$ will be different from $R_{R}$ if we have $x \eta_{2} \neq 0$. We have, in particular, $\lambda= \pm x \eta_{1}, T=e^{-2 x \eta_{1} L}, R_{L}=0$, and $R_{R}=\left[e^{-2 x \eta_{1} L}-\right.$ $1]^{2} / x^{2}$ at the exceptional point of $x=2 / \eta_{2}$. For sufficiently long and dense atomic lattices, we further have $T \rightarrow 0$ and $R_{R} \rightarrow 1 / x^{2}=\left[f_{p a}\left(b_{1}-b_{3}\right)\right]^{2} /\left[2 f_{p b}\left(b_{0}-\right.\right.$ $\left.\left.b_{2}\right)\right]^{2} \simeq\left[\left(b_{1}-b_{3}\right) /\left(b_{0}-b_{2}\right)\right]^{2} / 4$ so that a smaller $d / a$ is desired for getting a larger $R_{R}$. Modulating the dressing field detuning from $+\Delta_{d}$ to $-\Delta_{d}$, we have instead $T \sim R_{R} \rightarrow 0$ and $R_{L} \simeq\left[\left(b_{1}-b_{3}\right) /\left(b_{0}-b_{2}\right)\right]^{2} / 4$. This is the controlled unidirectional invisibility of realistic atomic lattices required by essential devices in optical networks like photonic diodes and transistors. Above predictions based on coupled-mode equations are in good agreement with numerical calculations based on transfer-matrix equations (cf. relevant curves in Figs. 5 and 6).

\section{IV. $P$ T-ANTISYMMETRIC SCATTERING}

The $P T$-antisymmetric optical lattices discussed in the previous sections can be probed in experiment via light scattering [1], which delivers comprehensive insights into their spectral properties and illuminates relevant consequences of the underlying physical symmetries which we now address. These properties can be encoded into the scattering matrix $S$, which relates the outgoing (electric-) field amplitudes $\left\{E_{\mathrm{L}}^{-}, E_{\mathrm{R}}^{+}\right\}$to the incoming (electric-) field amplitudes $\left\{E_{\mathrm{R}}^{-}, E_{\mathrm{L}}^{+}\right\}$ (see Fig. 1) as [37,38]

$$
\left(\begin{array}{c}
E_{\mathrm{L}}^{-} \\
E_{\mathrm{R}}^{+}
\end{array}\right)=S\left(\begin{array}{c}
E_{\mathrm{R}}^{-} \\
E_{\mathrm{L}}^{+}
\end{array}\right) \equiv\left(\begin{array}{c}
t_{\mathrm{R}} r_{\mathrm{L}} \\
r_{\mathrm{R}} t_{\mathrm{L}}
\end{array}\right)\left(\begin{array}{c}
E_{\mathrm{R}}^{-} \\
E_{\mathrm{L}}^{+}
\end{array}\right) .
$$

This relation is generally obtained by solving the coupled wave equations under appropriate boundary conditions in the scattering region, whose transmission and reflection properties are described by the complex amplitudes $\left\{t_{\mathrm{L}}, t_{\mathrm{R}}\right\}$ and $\left\{r_{L}, r_{R}\right\}$ respectively for transmission and reflection upon incidence from the left and from the right. For stationary optical lattices made of linear dielectric media with a symmetric susceptibility tensor, left to right transmission should always be equal to right to left transmission, i.e., $t_{\mathrm{L}}=t_{\mathrm{R}}=t$ (reciprocity), whereas only reflection shows handedness.
In general, the scattering matrix $S$ is non-Hermitian, its two eigenvalues

$$
\lambda_{s}^{ \pm}=t \pm \sqrt{r_{L} r_{R}}
$$

are complex, and the (unnormalized) eigenvectors $\left|\varphi_{ \pm}\right\rangle=$ $\left( \pm \sqrt{r_{L} / r_{R}}, 1\right)^{T}$ are not orthogonal. In particular, the two eigenvalues may merge into one another and the eigenvectors coalesce into a single one to yield a non-Hermitian $(\mathrm{NH})$ degeneracy [39,40], with the $S$ matrix being no longer diagonalizable. Such degeneracies occur either when $r_{\mathrm{L}}=0$ or when $r_{\mathrm{R}}=0$, and thus they are directly associated both with phase singularities in the geometric mean of reflection amplitudes $\left(r_{L}, r_{R}\right)$ and with unidirectional reflectionless propagation.

The phase-singular behavior of $\sqrt{r_{L} r_{R}}$ can be assessed by suitably rewriting $\lambda_{s}^{ \pm}-t$ in Eq. (12) in terms of the magnitude

$$
\left|\sqrt{r_{L} r_{R}}\right|=\left[\left(\operatorname{Re} \lambda_{s}^{ \pm}-\operatorname{Re} t\right)^{2}+\left(\operatorname{Im} \lambda_{s}^{ \pm}-\operatorname{Im} t\right)^{2}\right]^{1 / 2}
$$

and the phase

$$
\arg \sqrt{r_{L} r_{R}}=t g^{-1}\left[\frac{\operatorname{Im} \lambda_{s}^{+}-\operatorname{Im} t}{\operatorname{Re} \lambda_{s}^{+}-\operatorname{Re} t}\right] .
$$

The phase expression for the other eigenvalue is obtained upon replacing $\lambda_{s}^{+} \rightarrow \lambda_{s}^{-}$and adding an extra $\pi$ term on the righthand side of Eq. (14).

Typical patterns for $\operatorname{Im} \lambda_{s}^{ \pm}$and $\operatorname{Re} \lambda_{s}^{ \pm}$in our $P T$ antisymmetric optical lattices are plotted as a function of maximal dynamic shift $\delta_{d 0}$ in Figs. 7(a) and 7(b). The transition at $\delta_{d 0} \simeq 2.6 \mathrm{MHz} \rightarrow \delta_{0}^{N H}$ corresponds to a NH degeneracy $[39,40]$, where a pair of real eigenvalues degenerate and turn into complex conjugate pairs. This occurs when $\operatorname{Re} \lambda_{s}^{ \pm}=$ $\operatorname{Re} t \simeq|t|$ and $\operatorname{Im} \lambda_{s}^{ \pm}$vanishes [41]. More specifically, below degeneracy Eq. (13) reduces to

$$
\left|\sqrt{r_{L} r_{R}}\right| \simeq\left[\left(\operatorname{Re} \lambda_{s}^{ \pm}-|t|\right)^{2}+\operatorname{Im}^{2} \lambda_{s}^{ \pm}\right]^{1 / 2},
$$

with a similar expression holding above degeneracy provided $\operatorname{Im} \lambda_{s}^{ \pm} \rightarrow \operatorname{Im} t \operatorname{Re} \lambda_{s}^{+} / \operatorname{Re} t$, showing that $\left|\sqrt{r_{L} r_{R}}\right|$ vanishes almost continuously across degeneracy. Conversely, the reflection amplitudes $r_{L}$ and $r_{R}$ are in phase with the transmission amplitude $t$ for detunings above degeneracy but out of phase below degeneracy, i.e.,

$$
\begin{gathered}
\frac{\phi_{R}+\phi_{L}}{2}-\phi_{t} \simeq 0 \quad\left[\delta_{d 0}>\delta_{0}^{N H}\right], \\
\frac{\phi_{R}+\phi_{L}}{2}-\phi_{t} \simeq-\frac{\pi}{2} \quad\left[\delta_{d 0}<\delta_{0}^{N H}\right],
\end{gathered}
$$

where $\phi_{t}=\arg t$. This clearly arises from the behavior of the inverse tangent in Eq. (14) across degeneracy. Moving, e.g., along the $\lambda_{s}^{+}$(red circles) eigenvalue branch for values of detunings below $\delta_{0}^{N H}$ where $\operatorname{Im} t \ll \operatorname{Im} \lambda_{s}^{+}$[see Figs. 7(a) and $7(\mathrm{~g})]$, we can suitably rewrite Eq. (14) as

$$
\arg \sqrt{r_{L} r_{R}} \simeq \phi_{t}+t g^{-1}\left[\frac{\operatorname{Im} \lambda_{s}^{+}}{\operatorname{Re} \lambda_{s}^{+}-|t|}\right]
$$

while a similar expression holds above $\delta_{0}^{N H}$ where $\operatorname{Im} t>$ $\operatorname{Im} \lambda_{s}^{+} \sim 0$ instead [see Figs. 7(a) and 7(g)] provided $\operatorname{Im} \lambda_{s}^{+} \rightarrow$ $\operatorname{Im} t \operatorname{Re} \lambda_{s}^{+} / \operatorname{Re} t$. In this case, however, the inverse-tangent term in Eq. (18) is very small. Right at degeneracy the inversetangent term becomes undefined giving rise to the singular 

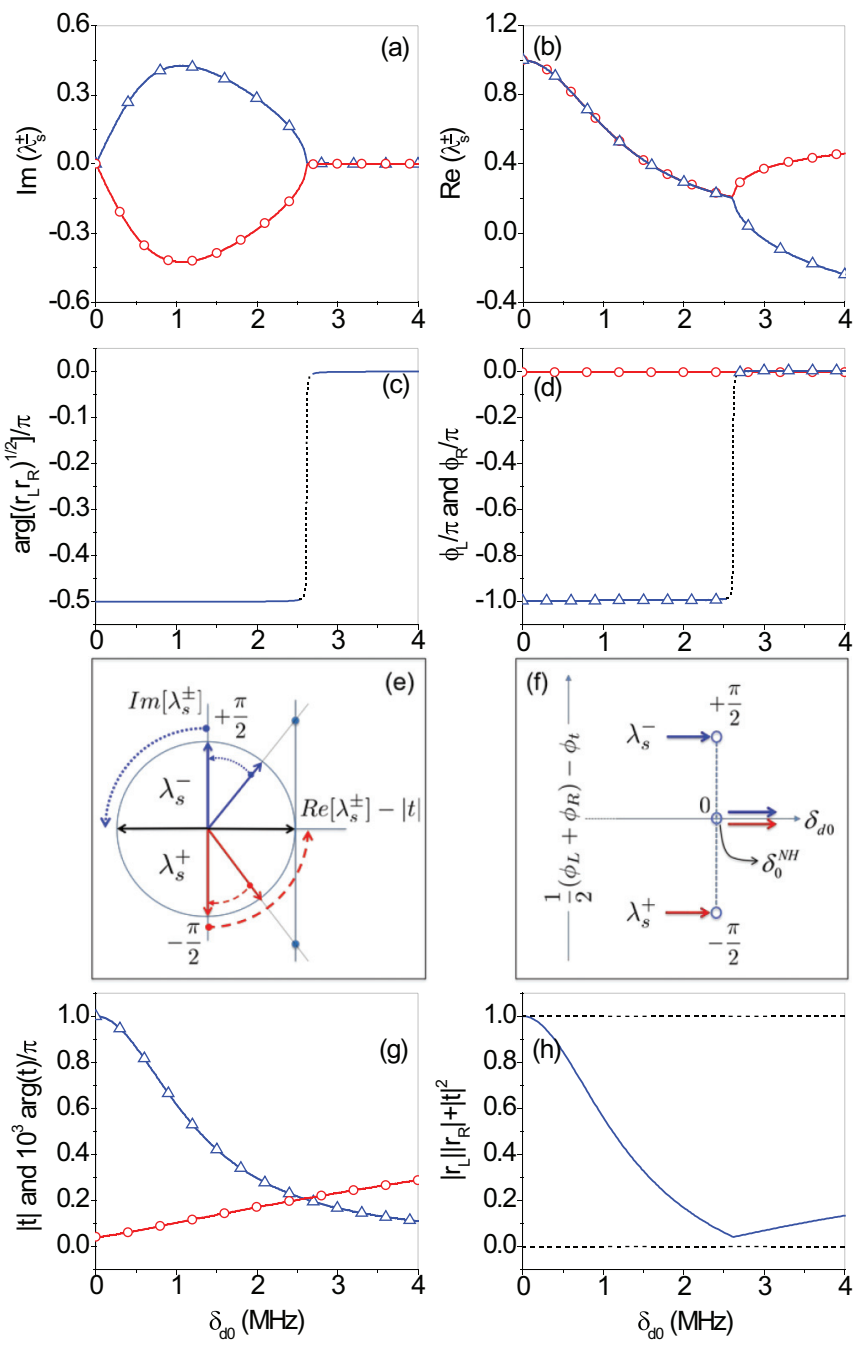

FIG. 7. (Color online) $\operatorname{Im}\left(\lambda_{s}^{ \pm}\right)$(a) and $\operatorname{Re}\left(\lambda_{s}^{ \pm}\right)$(b) of the scattering matrix eigenvalues vs maximal dynamic shift $\delta_{d 0}$. The red-circle and blue-triangle curves identify respectively $\lambda_{s}^{+}$and $\lambda_{s}^{-}$, showing a NH-degeneracy point at their crossing $\delta_{0}^{N H} \simeq 2.6 \mathrm{MHz}$. (c) The phase singularity of $\sqrt{r_{L} r_{R}}$ at $\delta_{0}^{N H}$ with (d) relevant phases $\phi_{L}$ (blue triangle) and $\phi_{R}$ (red circle) of the reflection amplitudes $r_{L}$ and $r_{R}$. (e) The red-dashed arrow (phasor) represents the jump of the inverse tangent in Eq. (18) while crossing degeneracy from below along the $\lambda_{s}^{+}$eigenvalue path. The blue-dotted arrow (phasor) shows a similar jump while crossing degeneracy along the $\lambda_{s}^{-}$ eigenvalue path. (f) Schematic of the phase jump at $\delta_{0}^{N H}$ along the two eigenvalue paths. (g) Magnitude (blue triangle) and phase (red circle) of the transmission amplitude $t$ as well as (h) the unitarity condition in Eq. (19) where unity (black dotted) represents unitarity. Relevant parameters are the same as in Fig. 5 except $\Delta_{p}=0$ and $a=10 d=400 \mathrm{~nm}$.

pattern of the $\arg \sqrt{r_{L} r_{R}}$ shown in Fig. 7(c). The corresponding $\pi / 2$ jump of the inverse tangent in Eq. (18) at degeneracy is further illustrated in Fig. 7(e). A similar discussion holds when crossing degeneracy along the $\lambda_{s}^{-}$(blue triangles) eigenvalue branch, yet yielding the same overall $\pi / 2$ shift in the phase of $\sqrt{r_{L} r_{R}}$ [see Fig. 7(f)] and hence the same phase relations in Eqs. (16) and (17). We also show in Fig. 7(d) the individual phases $\phi_{L}$ and $\phi_{R}$ of reflection amplitudes $r_{L}$ and $r_{R}$. It is clear that only $\phi_{L}$ is responsible for the $\pi$ jump across degeneracy while the latter remains there steady around $\phi_{R} \simeq-0.01 \times \pi$.

It is further worth noticing that Eqs. (16) and (17) are reminiscent of the phase relations in $P T$-symmetric photonic heterostructures with balanced gain and loss [42] where the phase jump corresponds to a phase singularity appearing at $|t|^{2}=1$, i.e., at the transition between loss and gain. In our purely lossy system, clearly not $P T$ symmetric, the phase jump is instead associated with a phase singularity appearing where $|t|^{2} \ll 1$, i.e., in a regime of remarkable loss. The phase relations in Eqs. (16) and (17) are also at variance with the corresponding phase relations in $P T$-antisymmetric heterostructures with balanced positive and negative index materials [29], whose amplitudes are subject to the restriction $\operatorname{Im} t=0$ and $r_{L}=r_{R}^{*}$. Our complex refractive index is not even $P T$ antisymmetric, with its real part always being positive.

Unitary conditions for our lossy system also bear some resemblance with those for $P T$-symmetric heterostructures. From Eq. (12), in fact, unitarity requires [43]

$$
\left|r_{L}\right|\left|r_{R}\right|=\left(|t|^{2}-1\right) e^{2 i\left[\phi_{t}-\left(\phi_{R}+\phi_{L}\right) / 2\right]}
$$

which is exactly the same generalized unitary relationship as given in Eq. (8) of Ref. [42]. Unitarity, clearly intrinsic to $P T$ symmetric systems [42], may instead be only observed below degeneracy, since $|t|^{2}<1$, in our passive $P T$-antisymmetric optical lattice. Departures from unitarity are in our case quantified in Fig. 7(h) where we have plotted $|t|^{2}+\left|r_{L}\right|\left|r_{R}\right|$. Notice, on the other hand, that for the case of $P T$-antisymmetric heterostructure with balanced positive and negative index materials [29] no phase relations come into play and the corresponding unitarity condition $\left|r_{L}\right|\left|r_{R}\right|=\left|r_{L}\right|^{2}=|t|^{2}-1$ is never satisfied in the passive regimes $\left(|t|^{2}<1\right)$. It should finally be emphasized at this stage that our passive half-mmlong $P T$-antisymmetric atomic lattice exhibits a rather sizable transmission with $|t| \simeq 0.2$ at degeneracy.

Non-Hermitian degeneracies are commonly described in terms of a two-dimensional parameter space where two eigenvalues pass through a branch-point singularity [30,39] showing a typical permutation of the eigenvalues if one encircles the singularity in this parameter space. The two eigenvalues represent the two branches of one analytic function exhibiting the singularity and are interchanged after one loop around the singularity, while away from the degeneracy the branching eigenvalues are different and each of them belongs to a distinct eigenvector. In matrix terms, at a NH-degeneracy point both eigenvalues belong to the same Jordan block and the $S$ matrix is not diagonalizable. Now, the permutation of the eigenvalues can be seen by computing them along a closed loop in the complex-parameter plane which in our case can be characterized by the two detunings $\Delta_{p}$ and $\delta_{d 0}$ combined into the complex parameter $z \equiv \Delta_{p}+i \delta_{d 0}$. Encircling the degeneracy at $\Delta_{p}=\Delta_{p}^{N H}=0$ and $\delta_{d 0}=\delta_{0}^{N H} \simeq 2.6 \mathrm{MHz}$ is achieved by letting $z \rightarrow \Delta_{p}^{N H}+i \delta_{0}^{N H}+\beta e^{i \theta}$, where the angle $\theta$ varies from 0 to $2 \pi$ while $z$ sweeps a circle of radius $\beta$. The situation is examined in Fig. 8 where phases of both reflection amplitudes and eigenvalues of the scattering matrix are plotted in the typical topology over one full encircling around the NH-degeneracy point. 

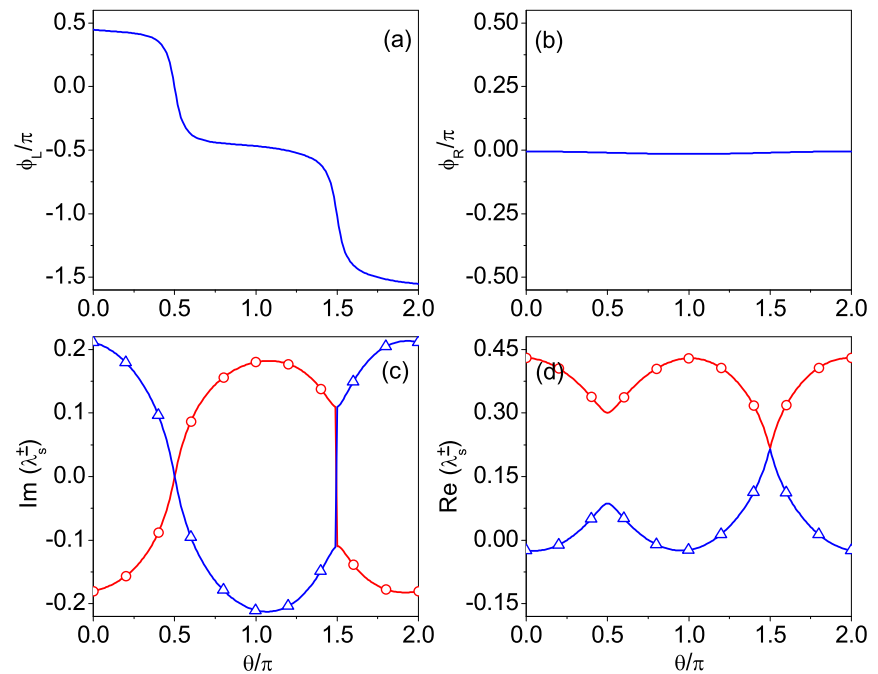

FIG. 8. (Color online) Plots of $\phi_{L}$ (a), $\phi_{R}$ (b), $\operatorname{Im}\left(\lambda_{s}^{ \pm}\right)$(c), and $\operatorname{Re}\left(\lambda_{s}^{ \pm}\right)(\mathrm{d})$ vs angle $\theta$ in the $\left\{\Delta_{p}, \delta_{d 0}\right\}$ space. Encircling occurs around the point $\left\{\Delta_{p}^{N H}=0, \delta_{0}^{N H}=2.6 \mathrm{MHz}\right\}$ with a radius $\beta=0.1 \mathrm{MHz}$. The red-circle and blue-triangle curves in panels $(\mathrm{c}, \mathrm{d})$ correspond, respectively, to $\lambda_{s}^{+}$and $\lambda_{s}^{-}$. Parameters are otherwise the same as in Fig. 6.

So far we have discussed NH degeneracies occurring at isolated points. As shown in Fig. 6, however, it may also be relevant to study the behavior of these degeneracies in the $\left(\Delta_{p}, \phi_{d}\right)$ parameter space where fully unidirectional reflection can be made to occur along continuous lines [44]. Figure 9 shows two lines of $\mathrm{NH}$ degeneracies in the $\left(\Delta_{p}, \phi_{d}\right)$ plane for $\delta_{d 0}=2.6 \mathrm{MHz}$. In a generic point in the $\left(\Delta_{p}, \phi_{d}\right)$ plane, the spatially dependent part of probe susceptibility to be used to set up the minimal coupled-mode model of Eqs. (9) converts to

$$
\begin{aligned}
\chi_{p j}(z) \rightarrow & \left(\chi_{c r}+i \chi_{c i}\right) \cos \left(2 k_{d} z\right) \\
& +\left(\chi_{s r}+i \chi_{s i}\right) \sin \left(2 k_{d} z\right)+\cdots ;
\end{aligned}
$$
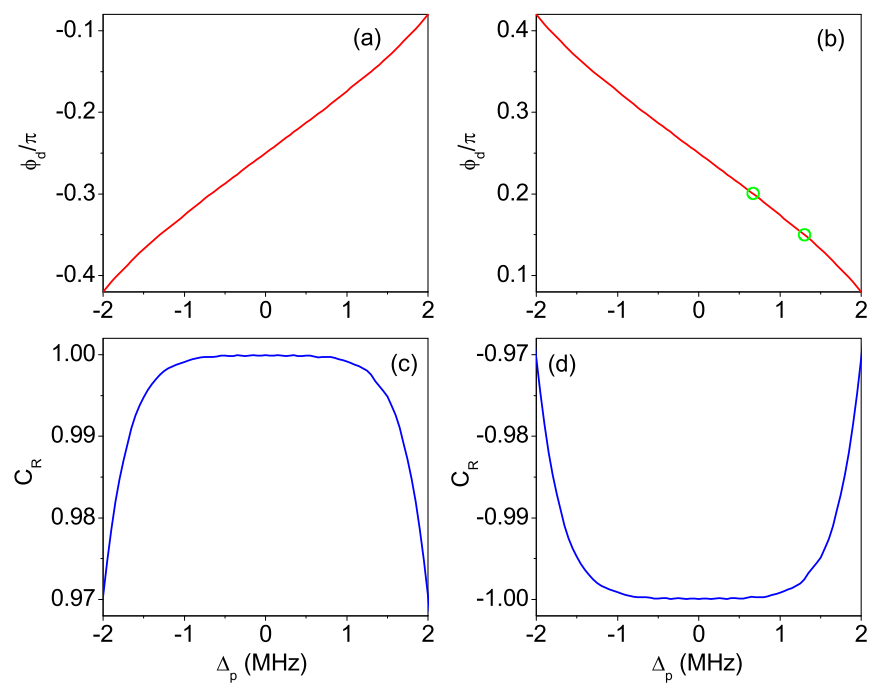

FIG. 9. (Color online) Lines of NH degeneracies in the $\left(\Delta_{p}, \phi_{d}\right)$ plane for a vanishing $R_{L}$ (a) and $R_{R}$ (b), with a reflectivity contrast $C_{R} \rightarrow+1.0$ (c) and $-1.0(\mathrm{~d})$, respectively. Parameters are otherwise the same as in Fig. 6.
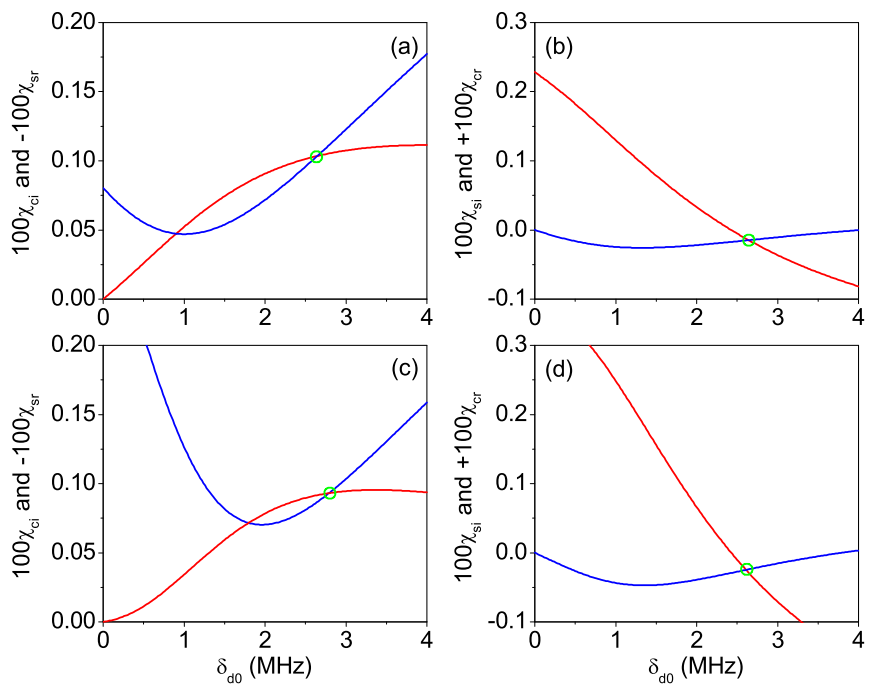

FIG. 10. (Color online) Conditions $\chi_{c r}=\chi_{s i}$ (symmetry) and $\chi_{c i}=-\chi_{s r}$ (antisymmetry) are satisfied accurately in panels $(\mathrm{a}, \mathrm{b})$ for $\left\{\Delta_{p}, \phi_{d}\right\}=\{0.68 \mathrm{MHz}, 0.2 \pi\}$ but approximately in panels (c, d) for $\left\{\Delta_{p}, \phi_{d}\right\}=\{1.30 \mathrm{MHz}, 0.15 \pi\}$. Parameters are otherwise the same as in Fig. 9.

along a NH-degeneracy line, however, the following two conditions $\chi_{c r}=\chi_{s i}$ (symmetry) and $\chi_{c i}=-\chi_{s r}$ (antisymmetry) are simultaneously satisfied, as plotted in Fig. 10 for two points (green circles) chosen from the degeneracy line in Fig. 9(b). Defining $\chi_{c r}=\chi_{s i} \equiv-\bar{\chi} \sin \alpha$ and $\chi_{c i}=-\chi_{s r} \equiv \bar{\chi} \cos \alpha$, we further attain

$\chi_{p j}(z) \rightarrow i \bar{\chi} \cos \left(2 k_{d} z+\alpha\right)-\bar{\chi} \sin \left(2 k_{d} z+\alpha\right)+\cdots$.

Obviously, in this case, the susceptibility profile is simply shifted along the lattice axis as compared to the case of Eq. (5b) $\left(\Delta_{p}=0\right)$ with $\chi_{p a}=\chi_{p b}$, which we recover for $\alpha \rightarrow 0$ and $\bar{\chi} \rightarrow \chi_{p a}=\chi_{p b}$, thus preserving fully unidirectional reflection. Note that for any values of $\alpha$ the real and imaginary parts of the spatially dependent susceptibility are out of phase with respect to one another. This is precisely what is required to ensure fully unidirectional reflection as recently discussed elsewhere.

\section{CONCLUSIONS}

In summary, we have designed a 1D optical lattice of cold atoms driven into a four-level $N$ configuration that exhibits perfect $P T$-antisymmetry in terms of probe susceptibility. This occurs when (i) ${ }^{87} \mathrm{Rb}$ atoms trapped in each dipole trap have a density distribution characterized by a cosine spatial modulation and when (ii) the dressing field is designed to generate a dynamic level shift exhibiting a sine spatial modulation [see Fig. 1(a)]. Such a $P T$-antisymmetric susceptibility yields high-contrast unbalanced reflectivities and unidirectional light invisibility at exceptional points where real and imaginary parts of the first-order susceptibility components have equal amplitudes and are out of phase. A detailed scattering matrix analysis shows that these points are NH degeneracies separating complex and real eigenvalues of the scattering matrix [45-48]. Unidirectional reflection corresponding to a reflection contrast $C_{R} \rightarrow \pm 1$ can be 
attained even when the dynamic level shift deviates from the (exact) sine modulation, that is, for a series of $\mathrm{NH}$ degeneracies lying on a line in the $\left(\Delta_{p}, \phi_{d}\right)$ plane. Measuring unidirectional light reflection from our $P T$-antisymmetric lattice provides a more viable tack to observing degeneracies of a non-Hermitian scattering matrix [13] and is clearly at variance with the more demanding techniques used, e.g., in coupled resonant cavities [49-51] where (eigen)frequencies rather than (reflected) intensities are in fact to be measured. The main advantage in terms of photonic applications is that in suitable media $P T$-antisymmetric susceptibility can be dynamically controlled, i.e., can be generated, tuned, and removed on demand. This is clearly a significant step forward as compared to the fixed $P T$ symmetry or antisymmetry of solid-state samples.

\section{ACKNOWLEDGMENTS}

This work is supported by the National Natural Science Foundation (Grants No. 11174110 and No. 61378094) of China and the National Basic Research Program (Grant No. 2011CB921603) of China. J.-H.W. would like to acknowledge the hospitality of Scuola Normale Superiore at Pisa.

\section{APPENDIX}

The $T W$ and $S W$ dressing components interact also with the $|1\rangle \leftrightarrow|3\rangle$ transition with relevant detunings (squared dipoles) being approximately 20 times (a half) of those on the $|2\rangle \leftrightarrow|4\rangle$ transition. Then corresponding level shifts amount to about $\delta_{d 0} / 40$, much smaller than the decay rate $\gamma$ and the trap depth $U_{o} \sim 2 \gamma$ (about 2000 times the recoil energy $E_{R}$ [52]). So we can safely neglect the spatial modulations of both atomic density $N_{0}$ and coupling detuning $\Delta_{c}$ due to this tiny level shift. In addition, the $T W$ dressing component traveling in the $x$ direction must have a linear polarization in the $y$ direction and is thus a superposition of plus and minus circular polarizations. The plus circular polarization couples the $|2\rangle \leftrightarrow|4\rangle$ transition as desired while the minus circular polarization couples wrongly the $|2\rangle \leftrightarrow|3\rangle$ transition. Fortunately, the level shift due to this wrong coupling is immaterial for our discussion because it is uniform in the $z$ direction and can be included into $\Delta_{c}$.

The light-shift difference $\delta \omega_{e g}$ of ground $5 S$ and excited $5 P_{1 / 2}$ states does matter in our scheme. But it can be reduced down to a negligible level by choosing for the lattice beams a suitable wavelength between the D1 and D2 lines. In fact, there is a magic wavelength $\sim 788 \mathrm{~nm}$ at which the ${ }^{87} \mathrm{Rb}$ atoms experience a vanishing $\delta \omega_{e g}$ on the D1 line [53]. Yet, we should also consider the light-shift difference $\delta \omega_{12}$ of ground $F=1$ and $F=2$ states. It is suppressed with respect to the trap depth $U_{o}$ by the ratio between the splitting of ground states and the detuning of lattice beams, i.e., about 1/400 leading to $\delta \omega_{12} \sim 20 \mathrm{kHz}$ negligible relative to $\delta_{d 0} \sim 2 \mathrm{MHz}$. But rather than $\delta \omega_{12}$ itself, its nonuniformity over the atomic distribution in a dipole trap should be considered. This amounts to an inhomogeneous contribution to the dephasing rate $\gamma_{12}$ on the Raman transition $|1\rangle \leftrightarrow|2\rangle$, which however does not alter our numerical results evidently.

The relative phase between $T W$ and $S W$ dressing components and the relative phase between lattice beams and dressing components should be accurately controlled. This is a very challenging task, but state-of-the-art techniques in cold atom physics [54] allow the accurate creation and manipulation of $1 \mathrm{D}, 2 \mathrm{D}$, and even $3 \mathrm{D}$ atomic lattices, which require indeed a good stabilization of the relative phases [55] between distinct counterpropagating lattice beams. For instance, in Ref. [56] a dichromatic optical lattice is obtained by superposing two periodic potentials of controllable intensities and phases, while in Ref. [57] a 2D optical lattice is realized by adjusting independently two relative phases $\theta$ and $\phi$.
[1] P. Markos and C. M. Soukoulis, Wave Propagation: From Electrons to Photonic Crystals and Left-Handed Materials (Princeton University Press, Princeton, NJ, 2008).

[2] E. Istrate and E. H. Sargent, Rev. Mod. Phys. 78, 455 (2006).

[3] D. R. Smith and N. Kroll, Phys. Rev. Lett. 85, 2933 (2000).

[4] A. Hache and M. Bourgeois, Appl. Phys. Lett. 77, 4089 (2000).

[5] X.-Y. Hu, Y.-H. Liu, J. Tian, B.-Y. Cheng, and D.-Z. Zhang, Appl. Phys. Lett. 86, 121102 (2005).

[6] X. Zhang and Z.-W. Liu, Nat. Mater. 7, 435 (2008).

[7] J. D. Teufel, T. Donner, M. A. Castellanos-Beltran, J. W. Harlow, and K. W. Lehnert, Nat. Nanotechnol. 4, 820 (2009).

[8] L. Chang, X.-S. Xiao, S.-Y. Hua, C. Yang, J.-M. Wen, L. Jiang, G.-Y. Li, G.-Z. Wang, and M. Xiao, Nat. Photon. 8, 524 (2014).

[9] B. Peng, S. K. Ozdemir, F.-C. Lei, F. Monifi, M. Gianfreda, G.-L. Long, S.-H. Fan, F. Nori, C. M. Bender, and L. Yang, Nat. Phys. 10, 394 (2014).

[10] D.-W. Wang, H.-T. Zhou, M.-J. Guo, J.-X. Zhang, J. Evers, and S.-Y. Zhu, Phys. Rev. Lett. 110, 093901 (2013).

[11] S. A. R. Horsley, J.-H. Wu, M. Artoni, and G. C. La Rocca, Phys. Rev. Lett. 110, 223602 (2013).
[12] Z. Lin, H. Ramezani, T. Eichelkraut, T. Kottos, H. Cao, and D. N. Christodoulides, Phys. Rev. Lett. 106, 213901 (2011).

[13] L. Feng, Y.-L. Xu, W. S. Fegadolli, M.-H. Lu, J. E. B. Oliveira, V. R. Almeida, Y.-F. Chen, and A. Scherer, Nat. Mater. 12, 108 (2012).

[14] C. M. Bender and S. Boettcher, Phys. Rev. Lett. 80, 5243 (1998).

[15] S. Longhi, Phys. Rev. Lett. 103, 123601 (2009).

[16] C. E. Ruter, K. G. Makris, R. EI-Ganainy, D. N. Christodoulides, M. Segev, and D. Kip, Nat. Phys. 6, 192 (2010).

[17] A. Regensburger, C. Bersch, M.-A. Miri, G. Onishchukov, D. N. Christodoulides, and U. Peschel, Nature (London) 488, 167 (2012).

[18] Y.-D. Chong, L. Ge, H. Cao, and A. D. Stone, Phys. Rev. Lett. 105, 053901 (2010).

[19] S. Longhi, Phys. Rev. A 82, 031801(R) (2010).

[20] N. I. Landy, S. Sajuyigbe, J. J. Mock, D. R. Smith, and W. J. Padilla, Phys. Rev. Lett. 100, 207402 (2008).

[21] V. V. Konotop, V. S. Shchesnovich, and D. A. Zezyulin, Phys. Lett. A 376, 2750 (2012). 
[22] M.-A. Miri, P. LiKamWa, and D. N. Christodoulides, Opt. Lett. 37, 764 (2012).

[23] F. Nazari, M. Nazari, and M. K. Moravvej-Farshi, Opt. Lett. 36, 4368 (2011).

[24] C. M. Bender, D. C. Brody, H. F. Jones, and B. K. Meister, Phys. Rev. Lett. 98, 040403 (2007).

[25] C. M. Bender, D. C. Bordy, J. Caldeira, U. Gunther, B. K. Meister, and B. F. Samsonov, Philos. Trans. R. Soc. A 371, 20120160 (2013).

[26] C. Hang, G.-X. Huang, and V. V. Konotop, Phys. Rev. Lett. 110, 083604 (2013).

[27] J.-T. Sheng, M.-A. Miri, D. N. Christodoulides, and M. Xiao, Phys. Rev. A 88, 041803(R) (2013).

[28] H.-J. Li, J.-P. Dou, and G.-X. Huang, Opt. Express 21, 32053 (2013).

[29] L. Ge and H. E. Tureci, Phys. Rev. A 88, 053810 (2013).

[30] J.-H. Wu, M. Artoni, and G. C. La Rocca, Phys. Rev. Lett. 113, 123004 (2014).

[31] A. Schilke, C. Zimmermann, P. W. Courteille, and W. Guerin, Phys. Rev. Lett. 106, 223903 (2011).

[32] A. Schilke, C. Zimmermann, and W. Guerin, Phys. Rev. A 86, 023809 (2012).

[33] J.-H. Wu, S. A. R. Horsley, M. Artoni, and G. C. La Rocca, Light Sci. Appl. 2, e54 (2013).

[34] J.-H. Wu, M. Artoni, and G. C. La Rocca, Phys. Rev. A 88, 043823 (2013).

[35] M. Fleischhauer, A. Imamoglu, and J. P. Marangos, Rev. Mod. Phys. 77, 633 (2005).

[36] M. Artoni, G. C. La Rocca, and F. Bassani, Phys. Rev. E 72, 046604 (2005).

[37] A. Mostafazadeh, Phys. Rev. Lett. 102, 220402 (2009).

[38] The $S$ matrix in Eq. (11) describes the input-output fields scattering geometry as sketched in Fig. 1(a). This scattering matrix conforms with the one adopted in Ref. [37] while slightly differing from, e.g., the one adopted in Eq. (1.10) of Ref. [1], where the two columns of $S$ and the two incoming field amplitudes are interchanged.

[39] M. Berry, Czech. J. Phys. 54, 1039 (2004).

[40] C. M. Bender, Rep. Prog. Phys. 70, 947 (2007).

[41] We have here $\operatorname{Im} t \ll \operatorname{Re} t \simeq|t|$ as shown in Fig. 7(g).

[42] L. Ge, Y. D. Chong, and A. D. Stone, Phys. Rev. A 85, 023802 (2012).

[43] From the theory of matrices it follows that $\operatorname{det} S=\lambda_{s}^{+} \lambda_{s}^{-}$and hence unitarity $(|\operatorname{det} S|=1)$ requires that Eq. (19) be satisfied.

[44] P. Ambichl, K. G. Makris, L. Ge, Y. Chong, A. D. Stone, and S. Rotter, Phys. Rev. X 3, 041030 (2013).

[45] T. Kato, Perturbation Theory of Linear Operators (Springer, Berlin, 1966).

[46] W. D. Heiss, Nucl. Phys. A 144, 417 (1970).

[47] W. D. Heiss, Phys. Rev. E 61, 929 (2000).

[48] N. F. Moiseyev and S. Friedland, Phys. Rev. A 22, 618 (1980).

[49] C. Dembowski, H.-D. Graf, H. L. Harney, A. Heine, W. D. Heiss, H. Rehfeld, and A. Richter, Phys. Rev. Lett. 86, 787 (2001).

[50] S. Klaiman, U. Gunther, and N. Moiseyev, Phys. Rev. Lett. 101, 080402 (2008).

[51] Q. H. Song and H. Cao, Phys. Rev. Lett. 105, 053902 (2010).

[52] G. Raithel, W. D. Phillips, and S. L. Rolston, Phys. Rev. Lett. 81, 3615 (1998).

[53] B. Arora, M. S. Safronova, and C. W. Clark, Phys. Rev. A 76, 052509 (2007).

[54] I. Bloch, Nat. Phys. 1, 23 (2005).

[55] N. V. Morrow, S. K. Dutta, and G. Raithel, Phys. Rev. Lett. 88, 093003 (2002).

[56] S. Folling, S. Trotzky, P. Cheinet, M. Feld, R. Saers, A. Widera, T. Muller, and I. Bloch, Nature (London) 448, 1029 (2007).

[57] L. Tarruell, D. Greif, T. Uehlinger, G. Jotzu, and T. Esslinger, Nature (London) 483, 302 (2012). 